\title{
Neuroprotective Effects of Bone Marrow Stromal Cell Transplantation in Combination With Treadmill Exercise Following Traumatic Brain Injury
}

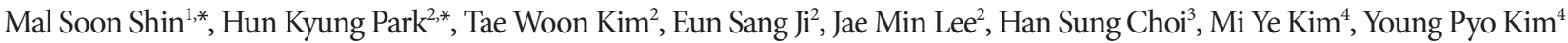 \\ ${ }^{1}$ School of Global Sport Studies, Korea University, Sejong, Korea \\ ${ }^{2}$ Department of Physiology, College of Medicine, Kyung Hee University, Seoul, Korea \\ ${ }^{3}$ Department of Emergency Medicine, College of Medicine, Kyung Hee University, Seoul, Korea \\ ${ }^{4}$ Department of Kinesiology, College of Natural Science, Jeju National University, Jeju, Korea
}

\begin{abstract}
Purpose: Traumatic brain injury (TBI) causes cognitive impairments, motor deficits, and neuropsychiatric/behavioral deficits problems. Transplantation of bone marrow stromal cells (BMSCs) facilitates functional recovery from brain insults. Treadmill exercise increases neurogenesis and inhibits apoptosis. In this study, we investigated the effects of BMSC transplantation in combination with treadmill exercise on memory function, by evaluating its effect on neurogenesis and apoptosis in the hippocampus following TBI.

Methods: TBI was induced using an electromagnetic-controlled cortical impact device. BMSCs were transplanted into both sides of traumatic scar region 1 week after TBI induction. One week after transplantation of BMSCs, the rats in the exercise groups were trained to run on a treadmill for 30 minutes once daily for 28 days. Step-down avoidance task and radial 8-arm maze test were conducted. Levels of 5-bromo-2'-deoxyuridine and caspase- 3 were evaluated using immunohistochemistry. Western blot was used to evaluate the expression of brain-derived neurotrophic factor (BDNF), tyrosine kinase B (TrkB), totalextracellular signal-regulated kinase 1 and 2 (t-ERK1/2), phosphorylated-ERK1/2 (p-ERK1/2), Bcl-2, and Bax.

Results: TBI deteriorated memory function, suppressed neurogenesis, and accelerated apoptosis in the hippocampus. Treadmill exercise and BMSC transplantation independently improved memory function by increasing neurogenesis with suppression of apoptosis through the BDNF-ERK pathway in the TBI-induced rats. Combination of BMSC transplantation with treadmill exercise showed additional enhancement of neurogenesis and suppression of apoptosis in the hippocampus. Conclusions: The present study shows that treadmill exercise may aid the therapeutic effect of BMSC transplantation on TBI in rats.
\end{abstract}

Keywords: Brain Injuries; Mesenchymal Stromal Cells; Exercise Test; Neurogenesis; Apoptosis

- Fund Support: This work was done with the sports promotion fund from Korea Institute of Sport Science, Seoul Olympic Sports Promotion Foundation (KISS-14-A05005).

- Conflict of Interest: No potential conflict of interest relevant to this article was reported.

Corresponding author: Young Pyo Kim (iD http://orcid.org/0000-0001-6662-1393 Department of Kinesiology, College of Natural Science, Jeju National University, 102 Jejudaehak-ro, Jeju 63243, Korea

E-mail: kimyp@jejunu.ac.kr / Tel: +82-64-754-3584 / Fax: +82-64-757-1752

${ }^{*}$ Mal Soon Shin and Hun Kyung Park contributed equally to this study as co-

first authors.

Submitted: May 1, 2016 / Accepted after revision: May 15, 2016
This is an Open Access article distributed under the terms of the Creative Commons Attribution Non-Commercial License (http://creativeses/by-nc/4.0/) which permits unrestricted non-commercial use, distribution, and reproduction in any medium, provided the original work is properly cited. 


\section{INTRODUCTION}

Traumatic brain injury (TBI) is one of the leading causes of neurological deficits, which result in cognitive impairments, motor deficits, and neuropsychiatric/behavioral deficits problems [1]. Various types of brain insults induce loss of neural precursor cells and reduce the number of newly generated neurons. These changes can result in deterioration of memory function [2] and inhibit long-term potentiation [3]. Neuronal loss has been suggested as the mechanism underlying the development of hippocampus-dependent cognitive deficits in TBI $[4,5]$.

Brain-derived neurotrophic factor (BDNF) acts via receptor tyrosine kinase $\mathrm{B}$ (TrkB), and the interaction of BDNF with TrkB is implicated in the synaptic plasticity, learning process, and neurogenesis [6,7]. BDNF activates TrkB signaling and its downstream effectors, including extracellular signal-regulated kinase (ERK). ERK 1 and 2 (ERK1/2) cascade promotes transcription of genes involving in the synapse formation [8].

TBI has been shown to cause apoptotic cell death in hippocampal neurons [4,9]. The members of the B-cell lymphoma 2 (Bcl-2) family and a class of cysteine proteases known as caspases are involved in the process of apoptotic cell death $[10,11]$. The $\mathrm{Bcl}-2$ family is divided into antiapoptotic proteins and pro-apoptotic proteins. Anti-apoptotic Bcl-2 protects against cell death by regulating apoptotic pathways. Pro-apoptotic Bax expression is enhanced during apoptosis, resulting in acceleration of cell death [10]. Decreased Bcl-2 immunoreactivity and increased Bax immunoreactivity were observed in the injured neurons after TBI [4] and global ischemia [12]. The most well-known member of the caspase family, caspase-3, plays a key role in apoptosis [11], and acts downstream of the Bax/Bcl-2 pathway [13].

Bone marrow stromal cells (BMSCs), also known as mesenchymal stem cells, are multipotent nonhematopoietic stem cells [14]. Transplantation of BMSCs facilitates regeneration of injured axons, and promotes functional recovery following central nervous system injuries via the differentiation of BMSCs into neurons [15-17]. In addition, treadmill exercise is also known to increase neurogenesis through enhancing neurotrophic factors, such as BDNF [7,18].

Transplantation of stromal cells has been used as one of the effective strategies for the treatment of TBI. However, the effects of treadmill exercise on BMSCs therapy in TBI have not been evaluated. In the present study, we investigated whether treadmill exercise potentiated the restorative effects of BMSC transplantation in rats with TBI-induced memory impairment, by evaluating their combined effects on neurogenesis and apoptosis in the hippocampus.

\section{MATERIALS AND METHODS}

\section{Cell Culture}

BMSCs were obtained from the femoral and tibial bones of 4-week-old rats, as previously described method [15]. Cells were cultured using $\alpha$-modified Eagle's medium ( $\alpha$-MEM) with $5 \% \mathrm{CO}_{2}$ at $37^{\circ} \mathrm{C}$ for 3 days. Subsequently, $10 \mu \mathrm{L}$ of BMSCs containing $10^{5}$ cells were injected into both sides of the traumatic scar region 1 week after TBI induction.

\section{Animals and Treatments}

Male Sprague-Dawley rats weighing $200 \pm 10 \mathrm{~g}$ (8 weeks) were purchased from the Orient Co. (Seoul, Korea). Experiments were performed in accordance with the guidelines of the Korean Academy of Medical Sciences and the animal care guidelines of the National Institutes of Health. The rats were divided into six groups ( $n=10$, each group): (1) control group, (2) treadmill exercise group, (3) TBI-induced group, (4) TBI-induced and treadmill exercise group, (5) TBI-induced and BMSC transplantation group, and (6) TBI-induced and BMSC transplantation with exercise group. One hour before starting treadmill running, the rats were injected subcutaneously with $50 \mathrm{mg} / \mathrm{kg}$ of 5-bromo-2'-deoxyuridine (BrdU, 50 mg/kg; Sigma Chemical Co., St. Louis, MO, USA), once a day for 4 consecutive days.

\section{Induction of TBI}

TBI was induced as previously described method [4,9]. The rats were anesthetized with Zoletil 50 (10 mg/kg, intraperitoneally; Virbac Laboratories, Carros, France). Contusion injury was created using an electromagnetic contusion device (Impact One, Stereotaxic Impactor; MyNeurolab, St. Louis, MO, USA) with a sterile stainless steel impactor tip (3.0 mm diameter) at a velocity of $5.00 \mathrm{~m} / \mathrm{sec}$. The rats in the control group and in the treadmill exercise group were treated in an identical manner, except that no brain contusion was simulated.

\section{Treadmill Exercise Protocol}

One week after BMSC transplantation, the rats in the exercise groups were trained to run on a treadmill for 30 minutes, once daily for 28 days. Exercise load consisted of running at a speed of $2 \mathrm{~m} / \mathrm{min}$ for the first 5 minutes, $5 \mathrm{~m} / \mathrm{min}$ for the next $5 \mathrm{~min}$ utes, and then at a speed of $8 \mathrm{~m} / \mathrm{min}$ for the last 20 minutes, at 
an inclination of $0^{\circ}$.

\section{Step-Down Avoidance Task}

Step-down avoidance task was used to evaluate short-term memory, as previously described method $[4,19]$. The rats were trained in a step-down avoidance task 42 days after TBI induction. In the training session, the animals received a scrambled foot shock of $0.5 \mathrm{~mA}$ for 2 seconds immediately upon stepping down. Two hours after training, the latency, defined as the interval between the rats stepping down and placing all four paws on the grid, was determined. Latency over 180 seconds is reported as 180 seconds.

\section{Radial 8-Arm Maze Test}

A radial 8-arm maze test was conducted for the evaluation of spatial learning ability, as previously described method [7,20]. The rats were trained three times before the test. After the training sessions, the rats were deprived of water for 24 hours. Subsequently, they were allowed to explore the maze to find the water and given 5 minutes to drink it. The test was conducted 41 days after TBI induction, and the test was terminated when the rats found water in all eight arms or when over 6 minutes had elapsed. Re-entry into the previously visited arms was counted as the error. The number of correct choices before the first error was also counted.

\section{Tissue Preparation}

The animals were sacrificed immediately after determining the latency in the step-down avoidance task. The rats were anesthetized using Zoletil 50 (10 mg/kg, intraperitoneally, Virbac Laboratories), followed by transcardial perfusion of $0.05 \mathrm{M}$ phosphate-buffered saline, and fixed using 4\% paraformaldehyde in $0.5 \mathrm{M}$ phosphate buffer ( $\mathrm{pH}$ 7.4). A freezing microtome (Leica, Nussloch, Germany) was used to prepare $40-\mu \mathrm{m}$ thick coronal sections.

\section{BrdU Immunohistochemistry}

BrdU-specific immunohistochemistry was performed as previously described method $[7,19]$. The sections were incubated overnight at $4^{\circ} \mathrm{C}$ with BrdU-specific mouse monoclonal antibody (1:600; Roche, Mannheim, Germany). Next, the sections were incubated with a biotinylated mouse secondary antibody (1:200; Vector Laboratories, Burlingame, CA, USA) for 1 hour. For visualization, the sections were incubated in $50 \mathrm{mM}$ Tris$\mathrm{HCl}$ (pH 7.6) containing $0.02 \%$ diaminobenzidine tetrahydro- chloride (DAB), $40 \mathrm{mg} / \mathrm{mL}$ nickel chloride, and $0.03 \% \mathrm{H}_{2} \mathrm{O}_{2}$ for 5 minutes. Differentiation of BrdU-positive cells was confirmed on the same section using a mouse antineuronal nuclei antibody (1:300; Chemicon International, Temecula, CA, USA).

\section{Caspase-3 Immunohistochemistry}

Caspase-3 immunohistochemistry was performed as previously described method $[4,20]$. The sections were incubated overnight with a rabbit anti-caspase-3 antibody (1:500; Santa Cruz Biotechnology, Dallas, TX, USA), and then incubated for another 1 hour with the biotinylated rabbit secondary antibody. The bound secondary antibody was then amplified using a Vector Elite ABC kit (Vector Laboratories). The antibody-biotinavidin-peroxidase complex was visualized using $0.02 \%$ DAB.

\section{Western Blot Analysis}

For the detection of the levels of BDNF, TrkB, total-ERK1/2 (tERK1/2), phosphorylated-ERK1/2 (p-ERK1/2), Bax, and Bcl-2, Western blot analysis was conducted as previously described method $[4,20]$. Hippocampal tissues were lysed in ice-cold lysate buffer. Proteins ( $40 \mu \mathrm{g})$ were separated on sodium dodecyl sulfate-polyacrylamide gels and transferred onto a nitrocellulose membrane (Amersham Pharmacia Biotechnology, Piscataway, NJ, USA). Mouse antiactin (1:500; Santa Cruz Biotechnology), rabbit anti-BDNF, anti-TrkB, anti-t-ERK1/2, anti-pERK1/2, anti-Bax, anti-Bcl-2 antibodies (1:1,000; Santa Cruz Biotechnology) were used as primary antibodies. Horseradish peroxidase-conjugated anti-mouse antibody for actin and antirabbit antibodies for BDNF, TrkB, t-ERK1/2, p-ERK1/2, and Bax, Bcl-2 were used as secondary antibodies. Protein bands were detected using the enhanced chemiluminescence detection system (Santa Cruz Biotechnology).

\section{Statistical Analysis}

Data were analyzed using IBM SPSS Statistics ver. 21.0 (IBM Co., Armonk, NY, USA). Results were expressed as the mean \pm standard error of the mean. One-way analysis of variance with Duncan post hoc test was used for comparison among the groups. $\mathrm{P}<0.05$ was considered statistically significant.

\section{RESULTS}

\section{Effects of BMSC Transplantation and Treadmill Exercise on Short-Term Memory}

Latency was significantly decreased by TBI induction. Tread- 
mill exercise and BMSC transplantation independently increased the latency in the TBI-induced rats. Combination of BMSC transplantation with treadmill exercise increased the la-

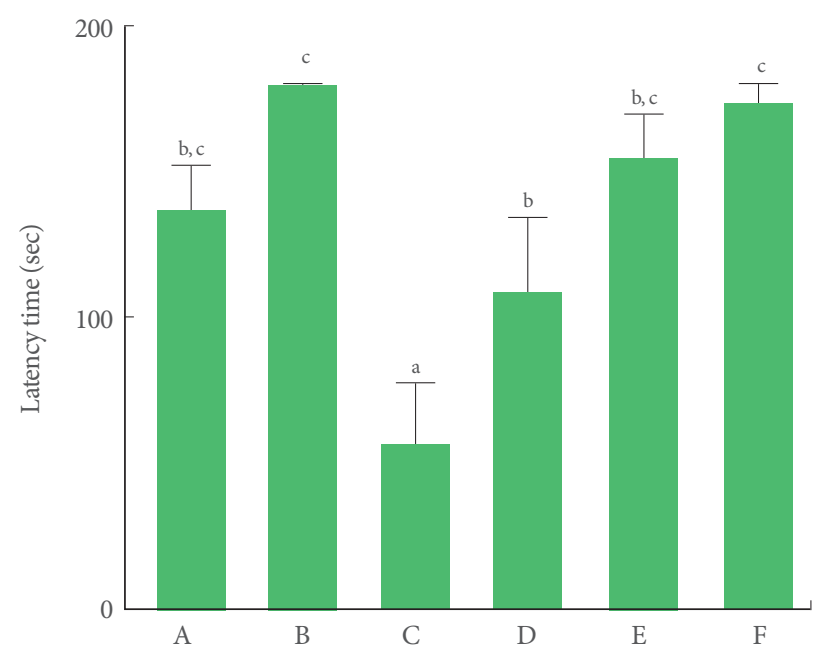

Fig. 1. Effects of transplantation of bone marrow stromal cells (BMSCs) and treadmill exercise on short-term memory. A, control group; $\mathrm{B}$, treadmill exercise group; $\mathrm{C}$, traumatic brain injury (TBI)-induced group; D, TBI-induced and treadmill exercise group; E, TBI-induced and BMSC transplantation group; F, TBI-induced and BMSC transplantation with treadmill exercise group. The letters a-c denote statistically significant differences, $\mathrm{P}<0.05$.

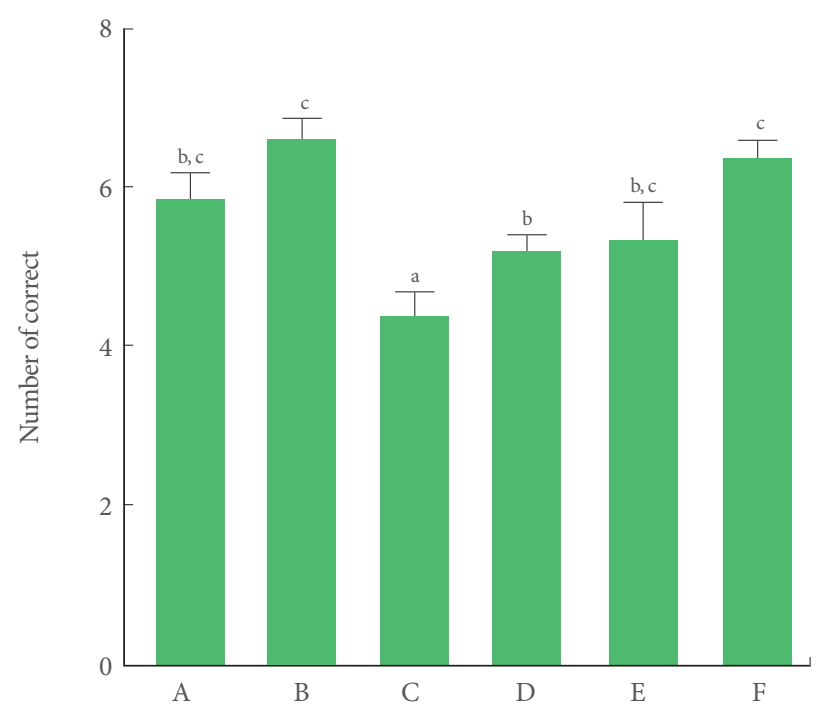

tency further, although the increase was not statistically significant (Fig. 1).

\section{Effects of BMSC Transplantation and Treadmill Exercise on Spatial Learning Ability}

On induction of TBI, the number of correct choices in the radial 8-arm maze test was significantly decreased and the number of errors was significantly increased. Treadmill exercise and BMSC transplantation independently increased the number of correct choices and decreased the number of errors in the TBIinduced rats. Combining BMSC transplantation with treadmill exercise had a potentiating effect on the number of correct choices; however, the increase in the number of correct choices did not reach statistical significance. In addition, there was no change in the number of errors in the combination protocol (Fig. 2).

\section{Effects of BMSC Transplantation and Treadmill Exercise on Neurogenesis in the Hippocampal Dentate Gyrus}

The number of BrdU-positive cells in the hippocampal dentate gyrus was significantly decreased by TBI induction. Treadmill exercise and BMSC transplantation independently increased the number of BrdU-positive cells in the TBI-induced rats, though there was no statistical significance. Combination of BMSC transplantation with treadmill exercise significantly in-

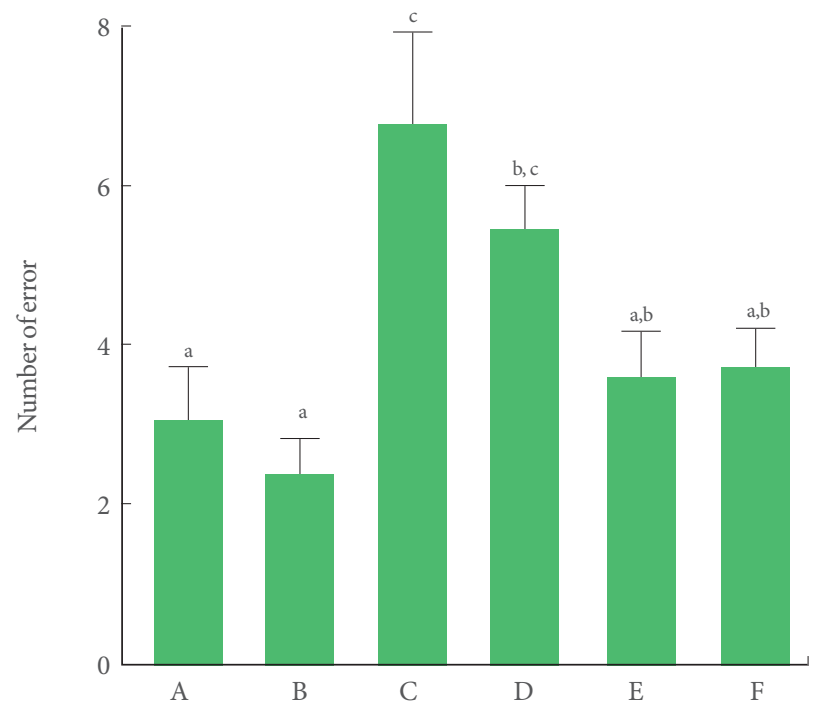

Fig. 2. Effects of transplantation of bone marrow stromal cells (BMSCs) and treadmill exercise on spatial learning ability. A, control group; B, treadmill exercise group; C, traumatic brain injury (TBI)-induced group; D, TBI-induced and treadmill exercise group; E, TBI-induced and BMSC transplantation group; F, TBI-induced and BMSC transplantation with treadmill exercise group. The letters a-c denote statistically significant differences, $\mathrm{P}<0.05$. 
creased the number of BrdU-positive cells (Fig. 3).

\section{Effects of BMSC Transplantation and Treadmill Exercise on Caspase-3 Expression in the Hippocampal Dentate Gyrus}

The number of caspase-3-positive cells in the hippocampal dentate gyrus was significantly increased by TBI induction. Treadmill exercise and BMSC transplantation independently decreased the number of caspase-3-positive cells in the TBI-induced rats. Combination of BMSC transplantation with treadmill exercise significantly decreased the number of caspase3-positive cells (Fig. 4).

\section{Effects of BMSC Transplantation and Treadmill Exercise on the BDNF-ERK Signaling Pathway}

BDNF and TrkB expression in the hippocampus was signifi-
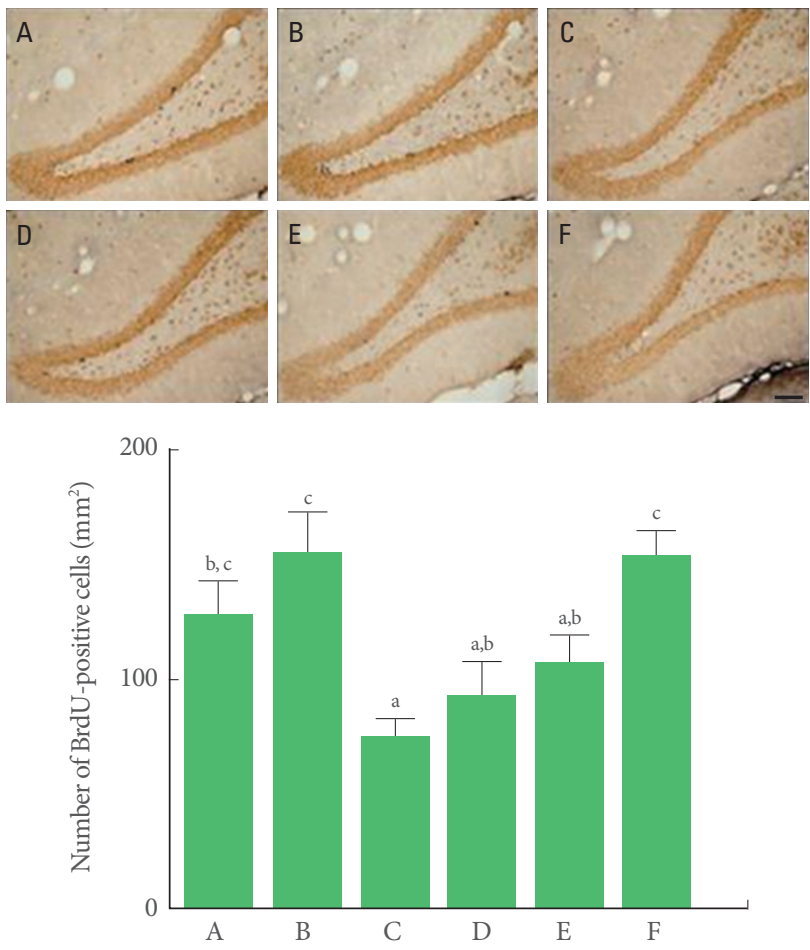

Fig. 3. Effects of transplantation of bone marrow stromal cells (BMSCs) and treadmill exercise on neurogenesis. Upper panel: Photomicrographs of 5-bromo-2'-deoxyuridine (BrdU)-positive cells. The scale bar represents $200 \mu \mathrm{m}$. Lower panel: Number of BrdU-positive cells in each group. A, control group; B, treadmill exercise group; $\mathrm{C}$, traumatic brain injury (TBI)-induced group; D, TBI-induced and treadmill exercise group; E, TBI-induced and BMSC transplantation group; F, TBI-induced and BMSC transplantation with treadmill exercise group. The letters a-c denote statistically significant differences, $\mathrm{P}<0.05$. cantly suppressed by TBI induction. Treadmill exercise and BMSC transplantation independently elevated BDNF and TrkB expression in the TBI-induced rats. Combination of BMSC transplantation with treadmill exercise significantly increased BDNF expression, but had no potentiating effect on TrkB expression. The ratio of $\mathrm{p}$-ERK to t-ERK in the hippocampus was significantly decreased by TBI induction. Treadmill exercise and BMSC transplantation independently increased this ratio in the TBI-induced rats. Combination of BMSC transplantation with treadmill exercise had no effect on this ratio (Fig. 5).

\section{Effects of BMSC Transplantation and Treadmill Exercise on Bax and $\mathrm{Bcl}-2$ Expressions}

Bax expression in the hippocampus was significantly increased and Bcl-2 expression in the hippocampus was significantly de-
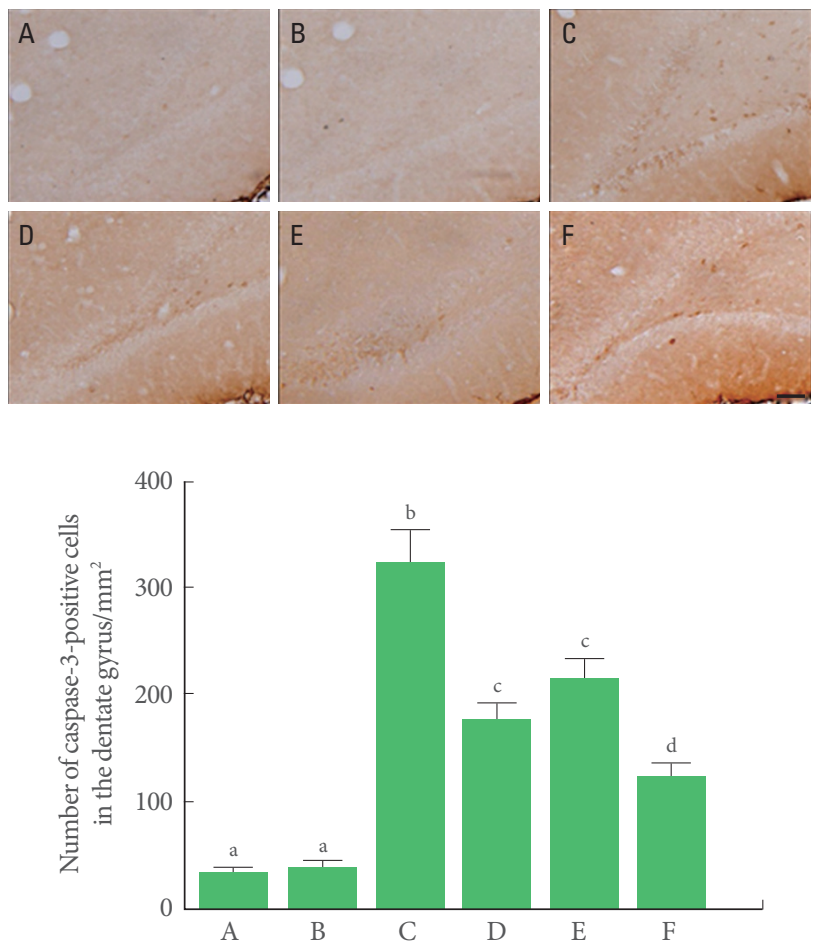

Fig. 4. Effects of transplantation of bone marrow stromal cells (BMSCs) and treadmill exercise on caspase-3 expression. Upper panel: Photomicrographs of caspase-3-positive cells. The scale bar represents $200 \mu \mathrm{m}$. Lower panel: Number of caspase-3-positive cells in each group. A, control group; $\mathrm{B}$, treadmill exercise group; C, traumatic brain injury (TBI)-induced group; D, TBIinduced and treadmill exercise group; E, TBI-induced and BMSC transplantation group; F, TBI-induced and BMSC transplantation with treadmill exercise group. The letters a- $d$ denote statistically significant differences, $\mathrm{P}<0.05$. 

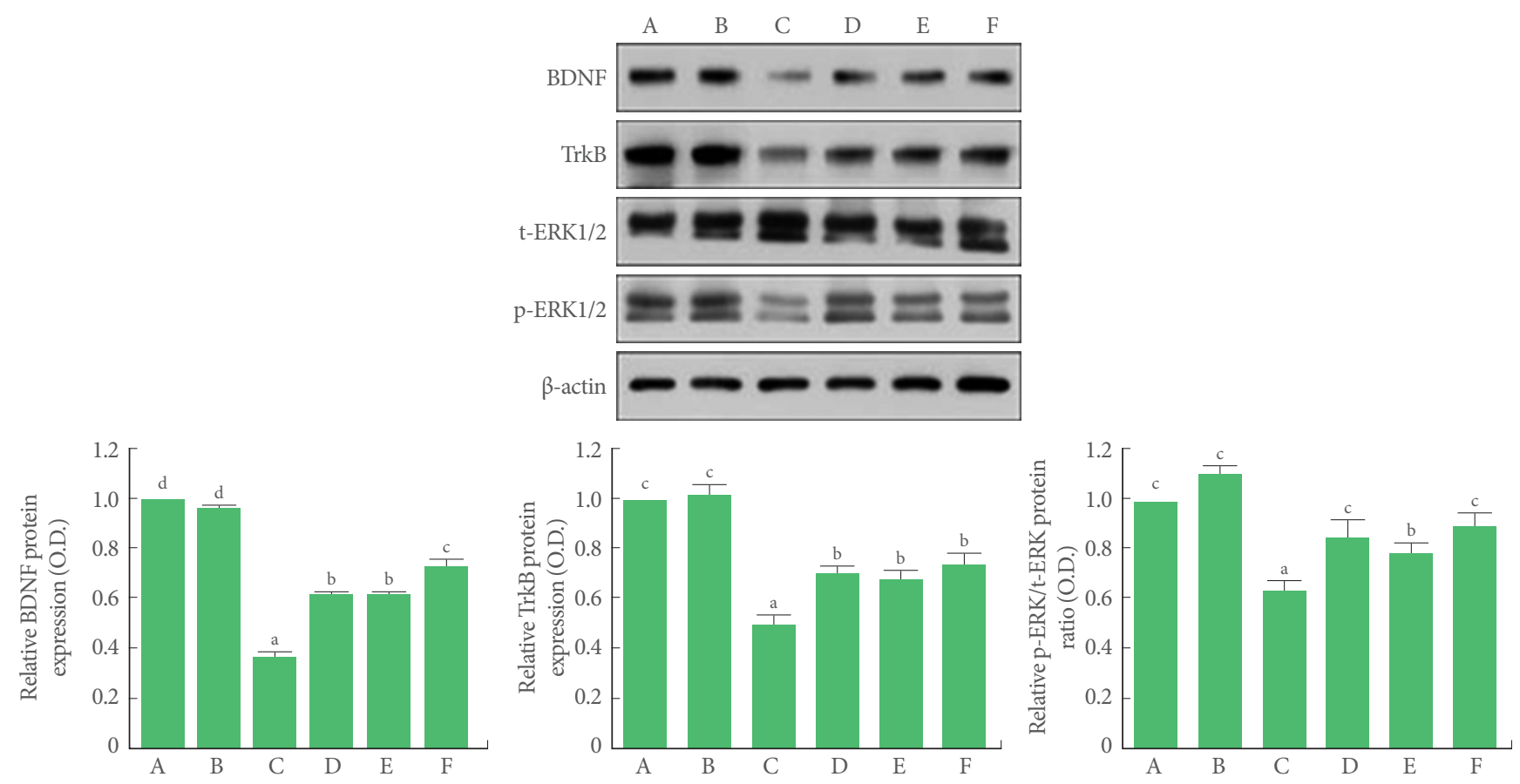

Fig. 5. Effects of transplantation of bone marrow stromal cells (BMSCs) and treadmill exercise on the expressions of brain-derived neurotrophic factor $(B D N F)$, tyrosine kinase B $(\operatorname{TrkB})$, phospho-extracellular signal-related kinase 1 and 2 (p-ERK1/2), and total extracellular signal-related kinase 1 and 2 (t-ERK1/2). A, control group; B, treadmill exercise group; C, traumatic brain injury (TBI)-induced group; D, TBI-induced and treadmill exercise group; E, TBI-induced and BMSC transplantation group; F, TBI-induced and BMSC transplantation with treadmill exercise group. The letters a-d denote statistically significant differences, $\mathrm{P}<0.05$.

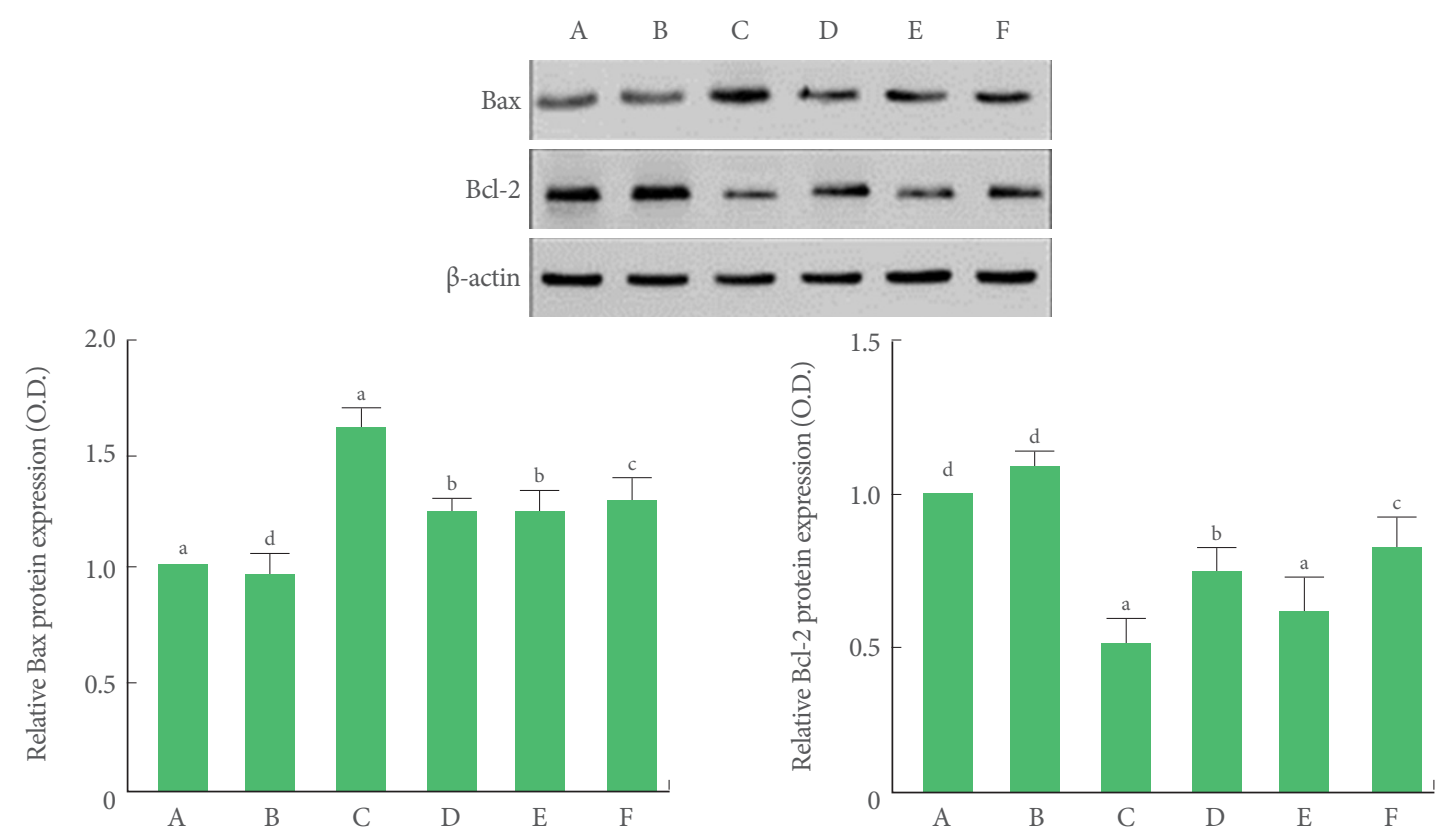

Fig. 6. Effects of transplantation of bone marrow stromal cells (BMSCs) and treadmill exercise on Bax and Bcl-2 expressions. (A) Control group, (B) treadmill exercise group, (C) TBI-induced group, (D) TBI-induced and treadmill exercise group, (E) TBI-induced and BMSC transplantation group, (F) TBI-induced and BMSC transplantation with treadmill exercise group. The letters a-d denote statistically significant differences, $\mathrm{P}<0.05$. 
creased by TBI induction. Treadmill exercise and BMSC transplantation independently decreased Bax expression in the TBIinduced rats. Treadmill exercise significantly increased Bcl-2 expression in the TBI-induced rats. Combination of BMSC transplantation with treadmill exercise did not decrease Bax expression, but significantly increased Bcl-2 expression (Fig. 6).

\section{DISCUSSION}

BMSC transplantation has been suggested as a useful strategy for cell-based therapy in stroke and TBI [21]. It promotes functional recovery by increasing endogenous neurogenesis after TBI [15]. Transplanted BMSCs were shown to survive, and improve neuronal behaviors in rats with TBI [17]. Treadmill exercise alleviated TBI-induced impairments in short-term memory and spatial learning ability by suppressing apoptosis in the hippocampus $[4,20]$. In this study, induction of TBI deteriorated short-term memory and spatial learning ability in rats. BMSC transplantation and treadmill exercise independently improved both short-term memory and spatial learning ability in the TBIinduced rats. We showed that transplantation of BMSCs has a stronger effect on improving spatial learning ability.

Reduction in the number of hippocampal neurons is associated with impaired performance in some hippocampus-dependent tasks [2]. Hippocampal neurogenesis is implicated in hippocampus-dependent learning ability $[2,22]$. The increasing effect of treadmill exercise on neurogenesis in the hippocampus has been well documented $[7,18]$ and BMSCs are known to possess the ability to promote endogenous repair mechanisms and to replace dying neurons [23]. In the current study, induction of TBI decreased hippocampal neurogenesis, and the combination of BMSC transplantation with treadmill exercise had a strong effect on increasing neurogenesis.

Exercise increases BDNF and TrkB expression, and subsequently the BDNF-TrkB signaling pathway promotes neurogenesis and inhibits apoptosis in the hippocampus [4,6,7]. ERK1/2 is activated by BDNF-TrkB signaling, and ERK1/2 activation mediates neurotropic actions such as neurogenesis [8,24]. BMSCs act as the resource for many trophic and growth factors that are implicated in differentiation, cell survival, and angiogenesis [25,26]. Treadmill exercise protects neurons against lipopolysaccharide exposure by increasing BDNF expression in the hippocampus [19]. Treadmill exercise enhanced BDNF expression in the hippocampus, which contributes to its anti-apoptotic effect [4]. Here we show that induction of TBI inhibited BDNF and
TrkB expression and inactivated the ERK pathway in the hippocampus. BMSC transplantation and treadmill exercise enhanced the expression of BDNF and TrkB, and as a result, activated the ERK1/2 pathway in the TBI-induced rats.

In our study, induction of TBI accelerated apoptosis in the hippocampus and TBI caused long-lasting cognitive impairment in rats [9]. Treadmill exercise and BMSC transplantation independently inhibited apoptosis in the TBI-induced rats. Combination of BMSC transplantation with treadmill exercise showed a stronger suppressing effect on apoptosis.

In the present study, combination of BMSC transplantation with treadmill exercise significantly increased neurogenesis and decreased apoptosis in the hippocampus. The present study shows that treadmill exercise may aid the therapeutic effect of BMSC transplantation on TBI.

\section{REFERENCES}

1. Tashlykov V, Katz Y, Gazit V, Zohar O, Schreiber S, Pick CG. Apoptotic changes in the cortex and hippocampus following minimal brain trauma in mice. Brain Res 2007;1130:197-205.

2. Shors TJ, Townsend DA, Zhao M, Kozorovitskiy Y, Gould E. Neurogenesis may relate to some but not all types of hippocampal-dependent learning. Hippocampus 2002;12:578-84.

3. Snyder JS, Kee N, Wojtowicz JM. Effects of adult neurogenesis on synaptic plasticity in the rat dentate gyrus. J Neurophysiol 2001;85: 2423-31.

4. Kim DH, Ko IG, Kim BK, Kim TW, Kim SE, Shin MS, et al. Treadmill exercise inhibits traumatic brain injury-induced hippocampal apoptosis. Physiol Behav 2010;101:660-5.

5. Witgen BM, Lifshitz J, Smith ML, Schwarzbach E, Liang SL, Grady MS, et al. Regional hippocampal alteration associated with cognitive deficit following experimental brain injury: a systems, network and cellular evaluation. Neuroscience 2005;133:1-15.

6. Chae $\mathrm{CH}$, Kim HT. Forced, moderate-intensity treadmill exercise suppresses apoptosis by increasing the level of NGF and stimulating phosphatidylinositol 3-kinase signaling in the hippocampus of induced aging rats. Neurochem Int 2009;55:208-13.

7. Kim DH, Ko IG, Kim BK, Kim TW, Kim SE, Shin MS, et al. Treadmill exercise and wheel exercise enhance expressions of neutrophic factors in the hippocampus of lipopolysaccharide-injected rats. Neurosci Lett 2013;538:54-9.

8. Ernfors P, Bramham CR. The coupling of a trkB tyrosine residue to LTP. Trends Neurosci 2003;26:171-3.

9. Park MS, Oh HA, Ko IG, Kim SE, Kim SH, Kim CJ, et al. Influence 
of mild traumatic brain injury during pediatric stage on short-term memory and hippocampal apoptosis in adult rats. J Exerc Rehabil 2014;10:148-54.

10. Kuwana T, Newmeyer DD. Bcl-2-family proteins and the role of mitochondria in apoptosis. Curr Opin Cell Biol 2003;15:691-9.

11. Cullen SP, Martin SJ. Caspase activation pathways: some recent progress. Cell Death Differ 2009;16:935-8.

12. Cho YS, Shin MS, Ko IG, Kim SE, Kim CJ, Sung YH, et al. Ulinastatin inhibits cerebral ischemia-induced apoptosis in the hippocampus of gerbils. Mol Med Rep 2015;12:1796-802.

13. Green D, Kroemer G. The central executioners of apoptosis: caspases or mitochondria? Trends Cell Biol 1998;8:267-71.

14. Javason EH, Colter DC, Schwarz EJ, Prockop DJ. Rat marrow stroma cells are more sensitive to plating density and expand more rapidly from single-cell-derived colonies than human marrow stromal cells. Stem Cells 2001;19:219-25.

15. Bonilla C, Zurita M, Otero L, Aguayo C, Vaquero J. Delayed intralesional transplantation of bone marrow stromal cells increases endogenous neurogenesis and promotes functional recovery after severe traumatic brain injury. Brain Inj 2009;23:760-9.

16. Neirinckx V, Agirman G, Coste C, Marquet A, Dion V, Rogister B, et al. Adult bone marrow mesenchymal and neural crest stem cells are chemoattractive and accelerate motor recovery in a mouse model of spinal cord injury. Stem Cell Res Ther 2015;6:211.

17. Shen Q, Yin Y, Xia QJ, Lin N, Wang YC, Liu J, et al. Bone marrow stromal cells promote neuronal restoration in rats with traumatic brain injury: involvement of GDNF regulating BAD and BAX signaling. Cell Physiol Biochem 2016;38:748-62.

18. Itoh T, Imano M, Nishida S, Tsubaki M, Hashimoto S, Ito A, et al.
Exercise increases neural stem cell proliferation surrounding the area of damage following rat traumatic brain injury. J Neural Trans 2011;118:193-202.

19. Kim K, Sung YH, Seo JH, Lee SW, Lim BV, Lee CY, et al. Effects of treadmill exercise-intensity on short-term memory in the rats born of the lipopolysaccharide-exposed maternal rats. J Exerc Rehabil 2015;11:296-302.

20. Ji ES, Kim YM, Shin MS, Kim CJ, Lee KS, Kim K, et al. Treadmill exercise enhances spatial learning ability through suppressing hippocampal apoptosis in Huntington's disease rats. J Exerc Rehabil 2015;11:133-9.

21. Li Y, Chopp M. Marrow stromal cell transplantation in stroke and traumatic brain injury. Neurosci Lett 2009;456:120-3.

22. O'Callaghan RM, Ohle R, Kelly AM. The effects of forced exercise on hippocampal plasticity in the rat: a comparison of LTP, spatialand non-spatial learning. Behav Brain Res 2007;176:362-6.

23. De Feo D, Merlini A, Laterza C, Martino G. Neural stem cell transplantation in central nervous system disorders: From cell replacement to neuroprotection. Curr Opin Neurol 2012;25:322-33.

24. Song HW, Kumar BK, Kim SH, Jeon YH, Lee YA, Lee WT, et al. Agmatine enhances neurogenesis by increasing ERK1/2 expression, and suppresses astrogenesis by decreasing BMP 2,4 and SMAD 1,5,8 expression in subventricular zone neural stem cells. Life Sci 2011;89:439-49.

25. Hess DC, Borlongan CV. Stem cells and neurological diseases. Cell Prolif 2008;41:94-114.

26. Jiang Y, Lv H, Huang S, Tan H, Zhang Y, Li H. Bone marrow mesenchymal stem cells can improve the motor function of a Huntington's disease rat model. Neurol Res 2011;33:331-7. 